Binghamton University

The Open Repository @ Binghamton (The ORB)

7-1-2019

\title{
Merging parallel-plate and levitation actuators to enable linearity and tunability in electrostatic MEMS
}

\author{
Mark Pallay \\ Ronald N. Miles \\ Shahrzad Towfighian \\ Binghamton University--SUNY, stowfigh@binghamton.edu
}

Follow this and additional works at: https://orb.binghamton.edu/mechanical_fac

Part of the Mechanical Engineering Commons, and the Physics Commons

\section{Recommended Citation}

Pallay, Mark; Miles, Ronald N.; and Towfighian, Shahrzad, "Merging parallel-plate and levitation actuators to enable linearity and tunability in electrostatic MEMS" (2019). Mechanical Engineering Faculty Scholarship. 24.

https://orb.binghamton.edu/mechanical_fac/24

This Article is brought to you for free and open access by the Mechanical Engineering at The Open Repository @ Binghamton (The ORB). It has been accepted for inclusion in Mechanical Engineering Faculty Scholarship by an authorized administrator of The Open Repository @ Binghamton (The ORB). For more information, please contact ORB@binghamton.edu. 


\title{
Merging parallel-plate and levitation actuators to enable linearity and tunability in electrostatic MEMS
}

\author{
Mark Pallay, ${ }^{1, a)}$ Ronald N. Miles, ${ }^{1, b)}$ and Shahrzad Towfighiann, ${ }^{1, c)}$ \\ Mechanical Engineering Department \\ Binghamton University \\ Binghamton, New York, 13902 USA
}

(Dated: 17 May 2019)

\begin{abstract}
In this study, a linear electrostatic MEMS actuator is introduced. The system consists of a MEMS cantilever beam with combined parallel-plate and electrostatic levitation forces. By using these two forcing methods simultaneously, the static response and natural frequency can be made to vary linearly with voltage. The static response shows a linear increase of $90 \mathrm{~nm}$ per volt and is maintained for more than $12 \mu$ m of tip displacement. The natural frequency shows a linear increase of $16 \mathrm{~Hz}$ per volt and is maintained throughout a $2.9 \mathrm{kHz}$ shift in natural frequency. This wide range of linear displacement and frequency tunability is extremely useful for MEMS sensors and actuators, which suffer from the inherent nonlinearity of electrostatic forces. A theoretical model of the system is derived and validated with experimental data. Static, natural frequency, and frequency response calculations are performed. Merging these two mechanisms enables high oscillation branches for a wide range of frequencies with potential applications in MEMS filters, oscillators and sensors.
\end{abstract}

\section{INTRODUCTION}

Nonlinearity in electrostatic microelectromechanical systems (MEMS) presents a challenging obstacle in the design of many MEMS sensors and actuators. Electrostatic MEMS convert electrical energy in the form of a voltage potential to kinetic and strain energy in a microstructure or vice versa. In most cases, MEMS are interfaced with electronic circuits to supply power or read the signal from a MEMS sensor. Ideally, sensors and actuators would have a linear relationship between voltage and the electrode position to simplify and miniaturize the electronic circuit. However, the electrostatic force is inherently nonlinear and typically has the relationship Force $\sim$ voltage $^{2} /$ gap $^{2}$, where the gap is the distance between electrodes. Nonlinearity effectively causes the devices to behave differently at different voltage levels and gaps, so the working range of a device is normally limited to small motions where the force can be treated as effectively linear. However, a smaller range creates less actuation distance for actuators and a smaller signal-tonoise ratio in sensors.

The most common structure of an electrostatic MEMS device consists of two electrodes that act like parallel plates. One electrode is fixed and the other is movable, such as a micro-beam that deflects under the influence of an external force. Applying a voltage potential between the two electrodes creates an electric field that pulls them together. This is the most common method of actuation and sensing in commercial MEMS because it has low bulk fabrication costs, very fast response times, and can be easily integrated into an electronic circuit ${ }^{1}$. However,

\footnotetext{
a)mpallay1@binghamton.edu

b)miles@binghamton.edu

c) stowfigh@binghamton.edu; Address all correspondence to this author.
}

the electrostatic force is highly nonlinear and can create instability that leads to pull-in (when the two electrodes collapse together), stiction ${ }^{2}$, and even chaos ${ }^{3}$. Alternative electrode arrangements have been proposed to avoid instability in electrostatic $\mathrm{MEMS}^{4-7}$, but all are hampered by the nonlinearity associated with electrostatic forces.

One alternative to parallel-plate actuation is electrostatic levitation ${ }^{8-13}$. The electrode configuration shown in Figure 1 creates an electric field that pushes electrodes apart instead of pulling them together. In this configuration, three electrodes are fixed to the substrate, and a beam is suspended above the center. The side electrodes are given a voltage relative to the common voltage of the center electrodes, which creates an electric field that wraps around and pulls on the top of the movable beam electrode more than the bottom, producing a net upward force. Much effort has been spent characterizing this system, and it has been shown to eliminate the pullin instability and increase travel ranges by more than an order of magnitude. However, this comes at the expense of requiring a large actuation voltage because the levitation force is relatively weak compared to the attractive force between a pair of parallel plates. The authors have experimentally shown this system can act as a switch by applying a bias voltage to the center electrode ${ }^{14}$. This allowed the switch to toggle to and from the pulled-in position for the purpose of creating a more durable switch. However, a comprehensive analysis of the system dynamics, including deriving a theoretical model, was not performed.

In this study, the parallel-plate and levitation mechanism is characterized by analyzing the static response, natural frequency, and frequency response. A theoretical model of the system is derived and validated with experimental data. We demonstrate the combined system can create a linear relationship with the side electrode voltage for the static response and first natural frequency. This 


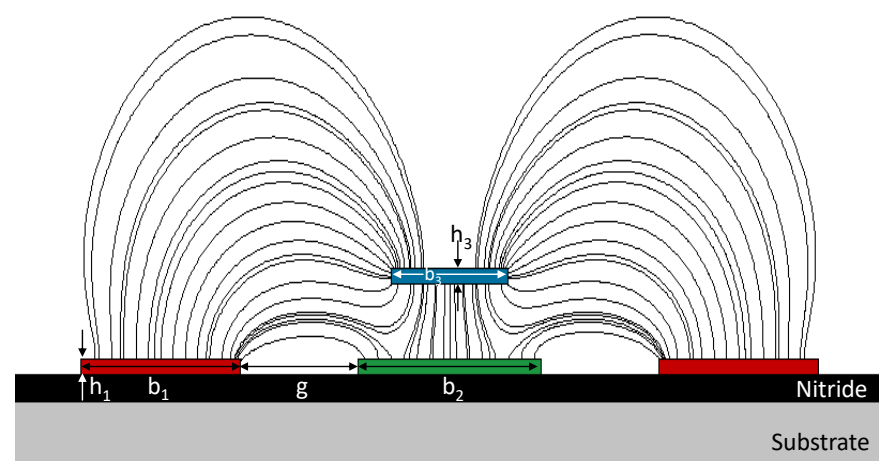

FIG. 1. Electrode layout to create electrostatic levitation with electric field lines. The side electrodes (red) are denoted as electrode 1 , the middle electrode (green) is denoted as electrode 2 , and the beam (blue) is denoted as electrode 3 . The geometric parameters $\left(h_{1}, b_{1}, g\right.$, etc.) are defined in Table II.

addresses the issues with the nonlinearity of electrostatic systems and can aid in the creation of highly tunable, linear, electrostatic MEMS with large travel ranges. The organization of this paper is as follows: the next section will outline the derivation of the theoretical model. Section III will outline the solution of the model and experimental results for the static, natural frequency, and frequency response.

\section{MODEL DERIVATION}

To model the actuator, the system can be treated as an Euler-Bernoulli beam with the governing equation of motion defined as,

$$
\rho A \frac{\partial^{2} \hat{w}}{\partial \hat{t}^{2}}+\hat{c} \frac{\partial \hat{w}}{\partial \hat{t}}+E I \frac{\partial^{4} \hat{w}}{\partial \hat{x}^{4}}=\hat{f}_{e}(\hat{w}, \vec{V})
$$

where $\hat{w}$ is the transverse beam deflection that depends on the position along the length of the beam, $x$, and time, $t$, and $\hat{f}_{e}(\hat{w}, \vec{V})$ is the electrostatic force, which depends on the transverse deflection and applied voltages. Because there are multiple electrodes with different voltages, $\vec{V}$ is a vector of voltages that are applied to the beam, side electrodes, and center electrode. The dimensions and material properties of the beam are given in Table I.

For a classic electrostatic beam with a parallel-plate electrode configuration, $\hat{f}_{e}$ can be represented analytically with an inverse polynomial of order two that has a singularity when the gap between electrodes goes to zero. However, in the system examined here, the simple analytical expression for the electrostatic force is not valid because of the different electrode arrangement, and the force must be calculated numerically. One way this can be achieved is to calculate the potential energy of the beam at each position, then take the derivative with
TABLE I. Beam parameters as shown in Figure 1

\begin{tabular}{lrr}
\hline \hline Parameter & Variable & Value \\
Beam Length & $\mathrm{L}$ & $505 \mu \mathrm{m}$ \\
Beam Width & $\mathrm{b}_{3}$ & $20.5 \mu \mathrm{m}$ \\
Beam Thickness & $\mathrm{h}_{3}$ & $2 \mu \mathrm{m}$ \\
Beam Anchor Height & $\mathrm{d}$ & $2 \mu \mathrm{m}$ \\
Side Electrode Gap & $\mathrm{g}$ & $20.75 \mu \mathrm{m}$ \\
Middle Electrode Width & $\mathrm{b}_{2}$ & $32 \mu \mathrm{m}$ \\
Side Electrode Width & $\mathrm{b}_{1}$ & $28 \mu \mathrm{m}$ \\
Electrode Thickness & $\mathrm{h}_{1}$ & $0.5 \mu \mathrm{m}$ \\
Dimple Length & $\mathrm{L}_{\mathrm{d}}$ & $0.75 \mu \mathrm{m}$ \\
Elastic Modulus & $\mathrm{E}$ & $160 \mathrm{GPa}$ \\
Density & $\rho$ & $2330 \mathrm{~kg} / \mathrm{m}^{3}$ \\
Poisson's Ratio & $\mathrm{v}$ & 0.22 \\
\hline \hline
\end{tabular}

respect to the direction of motion $(\hat{w})$. In this case, the potential energy is calculated from the voltage vector and capacitance matrix, the latter of which is simulated with a 2D finite-element analysis in COMSOL. The potential energy can be represented by,

$$
U=\frac{1}{2}\left[\begin{array}{lll}
V_{\text {side }} & V_{\text {bias }} & V_{\text {beam }}
\end{array}\right]\left[\begin{array}{lll}
\hat{c}_{11} & \hat{c}_{12} & \hat{c}_{13} \\
\hat{c}_{21} & \hat{c}_{22} & \hat{c}_{23} \\
\hat{c}_{31} & \hat{c}_{32} & \hat{c}_{33}
\end{array}\right]\left[\begin{array}{c}
V_{\text {side }} \\
V_{\text {bias }} \\
V_{\text {beam }}
\end{array}\right]
$$

where $\hat{c}_{i j}$ are the capacitances between each pair of electrodes, and $V_{\text {side }}, V_{\text {bias }}$, and $V_{\text {beam }}$, are the voltages on the side electrodes (1), center electrode (2), and beam (3) respectively. The capacitance matrix is symmetric with $c_{i j}=c_{j i}$, which results in 6 independent capacitances in the system. Expanding Equation (2), setting $V_{\text {beam }}=0$ because the beam is assumed to be the reference ground voltage level, and taking the derivative with respect to the transverse beam deflection, yields,

$$
\begin{aligned}
& \hat{f}_{e}(\hat{w}, \vec{V})= \\
& \quad \frac{1}{2}\left(V_{\text {side }}^{2} \frac{\partial \hat{c}_{11}}{\partial \hat{w}}+2 V_{\text {side }} V_{\text {bias }} \frac{\partial \hat{c}_{12}}{\partial \hat{w}}+V_{\text {bias }}^{2} \frac{\partial \hat{c}_{22}}{\partial \hat{w}}\right)
\end{aligned}
$$

In Equation (3), the derivatives of the COMSOL simulated capacitance, $\hat{c}_{i j}$, can be estimated with a simple central difference method. $\frac{\partial \hat{c}_{11}}{\partial \hat{w}}$ and $\frac{\partial \hat{c}_{12}}{\partial \hat{w}}$ can be fit with 9th order polynomials to create an analytical representation of the numerical data that can be used in Equation (1). $\frac{\partial \hat{c}_{22}}{\partial \hat{w}}$, however, would require upward of a 20 th order polynomial for an adequate fit, and therefore requires more consideration regarding its representation in Equation (1). We will treat this term differently for the static, natural frequency, and frequency response calculations, which will be discussed in the next section. For now, it will be left as a variable. 
TABLE II. Nondimensional substitutions and forcing coefficients for the governing differential equation

\begin{tabular}{lr}
\hline \hline Parameter & Substitution \\
x-direction position & $x=\hat{x} / L$ \\
z-direction position & $w=\hat{w} / h_{3}$ \\
Time & $t=\hat{t} / T$ \\
Capacitance & $c_{22}=\hat{c}_{22} / c_{n}$ \\
Time constant & $T=\sqrt{\rho A L^{4} / E I}$ \\
Damping Constant & $c^{*}=\hat{c} / E I T$ \\
Capacitance Constant & $c_{n}=\rho A L h_{3}^{2} / L^{2} T^{2}$ \\
Force Constant 1 & $A_{i}=\alpha_{i} h_{3}^{i-1} L^{4} / 2 E I$ \\
Force Constant 2 & $B_{i}=\beta_{i} h_{3}^{i-1} L^{4} / E I$ \\
\hline \hline
\end{tabular}

Figure 2 shows the three capacitances from the finite element solution (COMSOL) and the electrostatic force at various side and bias voltages. From Equation (3), when the bias voltage is set to zero, the only remaining force component is $\frac{\partial \hat{c}_{11}}{\partial \hat{w}}$, which represents the electrostatic levitation force. Conversely, if the side voltage is set to zero, only $\frac{\partial \hat{c}_{22}}{\partial \hat{w}}$ remains and the system acts as a parallel-plate. By observing the $c_{11}$ and $c_{22}$ capacitances, it is clear that $c_{22}$ has a much larger nominal capacitance and the magnitude of the slope is greater, especially at small gaps. This shows the parallel-plate force from the bias voltage is by far the dominant force component in this system. To counter this, much larger side voltages are needed to overcome the effect of the bias voltage. Figure 2d shows the calculated force for several voltage cases. At large gaps, the force is dominated by the levitation force, but as the gap decreases, the total force becomes strongly negative as the behavior is dominated by the attractive parallel-plate force, despite the side voltage being an order of magnitude larger than the bias voltage.

After the force is calculated, Equation (3) is plugged into Equation (1) yielding the dimensionalized equation of motion for the cantilever,

$$
\begin{array}{r}
\rho A \frac{\partial^{2} \hat{w}}{\partial \hat{t}^{2}}+\hat{c} \frac{\partial \hat{w}}{\partial \hat{t}}+E I \frac{\partial^{4} \hat{w}}{\partial \hat{x}^{4}}=\frac{1}{2} V_{\text {side }}^{2} \sum_{i=0}^{9} \alpha_{i} \hat{w}^{i}+ \\
V_{\text {side }} V_{\text {bias }} \sum_{i=0}^{9} \beta_{i} \hat{w}^{i}+\frac{1}{2} V_{\text {bias }}^{2} \frac{\partial \hat{c}_{22}}{\partial \hat{w}}
\end{array}
$$

where $\alpha_{i}$ and $\beta_{i}$ are fitting coefficients for the $c_{11}$ and $c_{12}$ terms respectively. Equation (4) is then nondimensionalized with the substitutions shown in Table II, which yields the final nondimensional equation of motion shown in Equation (5).

$$
\begin{aligned}
\frac{\partial^{2} w}{\partial t^{2}}+c^{*} \frac{\partial w}{\partial t}+ & \frac{\partial^{4} w}{\partial x^{4}}=V_{\text {side }}^{2} \sum_{i=0}^{9} A_{i} w^{i}+ \\
& V_{\text {side }} V_{\text {bias }} \sum_{i=0}^{9} B_{i} w^{i}+\frac{1}{2} V_{\text {bias }}^{2} \frac{\partial c_{22}}{\partial w}
\end{aligned}
$$

\section{RESULTS AND DISCUSSION}

\section{A. Static}

When the time-derivative terms in Equation (5) are set to zero, it becomes a fourth-order ordinary differential equation, with $x$ being the only independent variable. Despite the complicated forcing term, this equation can be solved directly as a boundary value problem. First, however, a form for $\frac{\partial \hat{c}_{22}}{\partial \hat{w}}$ must be determined. Because this force component behaves similarly to the parallelplate force, a fitting function with a similar form can be used. $\frac{\partial \hat{c}_{22}}{\partial \hat{w}}$ is fit with the function shown in Equation (6).

$$
\frac{\partial c_{22}}{\partial w}=\frac{-0.9252}{\left(w+\frac{d}{h_{3}}\right)^{2.15}}
$$

Plugging this into Equation (5) after dropping the time derivatives gives the static equation of motion that can be solved with the boundary value solver, bvp $4 c$, in MATLAB.

$$
\begin{aligned}
\frac{\partial^{4} w}{\partial x^{4}}= & V_{\text {side }}^{2} \sum_{i=0}^{9} A_{i} w^{i}+ \\
& V_{\text {side }} V_{\text {bias }} \sum_{i=0}^{9} B_{i} w^{i}-V_{\text {bias }}^{2} \frac{0.4626}{\left(w+\frac{d}{h_{3}}\right)^{2.15}}
\end{aligned}
$$

For the experiment, cantilevers with the electrode arrangement in Figure 1 are fabricated with the PolyMUMPs service by MEMSCAP ${ }^{15}$ to the dimensions given in Table I. An image of a fabricated beam is shown in Figure 3. Beam tip displacement is measured with a Polytec MSA-500 Laser vibrometer. A USB-6366 National Instruments Data Acquisition (DAQ) interfaced with MATLAB supplies the bias and side voltage and records the displacement data from the laser. Because the voltage limitation of the DAQ is +/- $10 \mathrm{~V}$, the side voltage is fed into a Krohn-Hite 7600 Wideband Power Amplifier and stepped up 20x to reach the desired 200V maximum voltage to the side electrodes. To measure the side voltage, the output of the amplifier is also fed into a resistive voltage divider to drop the voltage back down under $10 \mathrm{~V}$ and is fed back into the DAQ. The actual voltage on the side electrodes can be calculated with the sim- 

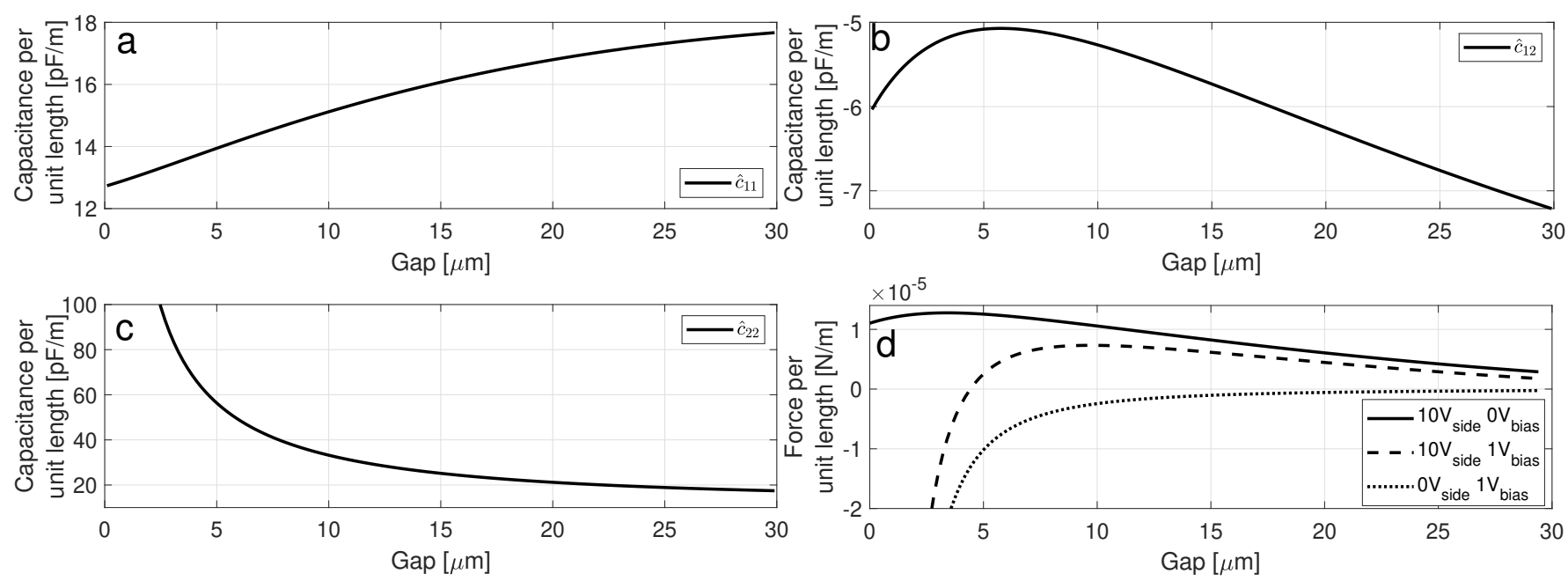

FIG. 2. a)-c) Capacitance per unit length of $\hat{c}_{11}, \hat{c}_{12}$, and $\hat{c}_{22}$ as calculated in COMSOL. d) Electrostatic force at various voltages showing the comparison between the pure attractive, pure repulsive, and combined forcing cases.

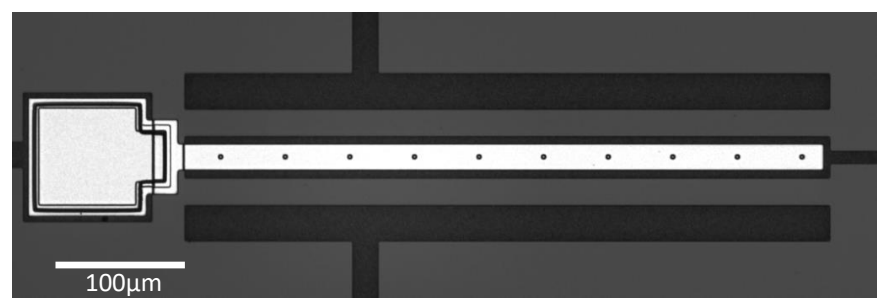

FIG. 3. Optical image of a fabricated beam.

ple voltage divider relationship and the DAQ measurement. A schematic of the experimental setup is shown in Figure 4.

In the experiment, the DAQ supplies a constant bias voltage to the center electrode and a quasi-static ramp function from $0 \mathrm{~V}$ to approximately $190 \mathrm{~V}$ to the side electrodes. The ramp duration is one second, which is four orders of magnitude longer than the natural frequency of the beam to ensure the beam response is quasi-static.

Figure 5 shows that the model agrees very well with the experiment, especially at higher bias voltages. To account for support compliance at the fixed end of the cantilever, a corrected length of $505 \mu \mathrm{m}$ is used. The tip displacement reaches almost $14 \mu \mathrm{m}$ at $190 \mathrm{~V}$, despite the anchor height of just $2 \mu \mathrm{m}$. These large travel ranges are not possible in conventional parallel plate actuators and are a distinct advantage of electrostatic levitation. For $4 \mathrm{~V}$ and $6 \mathrm{~V}$ bias, the beam pulls in if the side voltage is too low. In the experiment, the beam pulled in below a side voltage of approximately $50 \mathrm{~V}$ for $4 \mathrm{~V}$ bias, and $80 \mathrm{~V}$ for a $6 \mathrm{~V}$ bias. However, as noted in a previous experiment by the authors, when pull-in occurs, the side voltage can be ramped up to release the beam from its

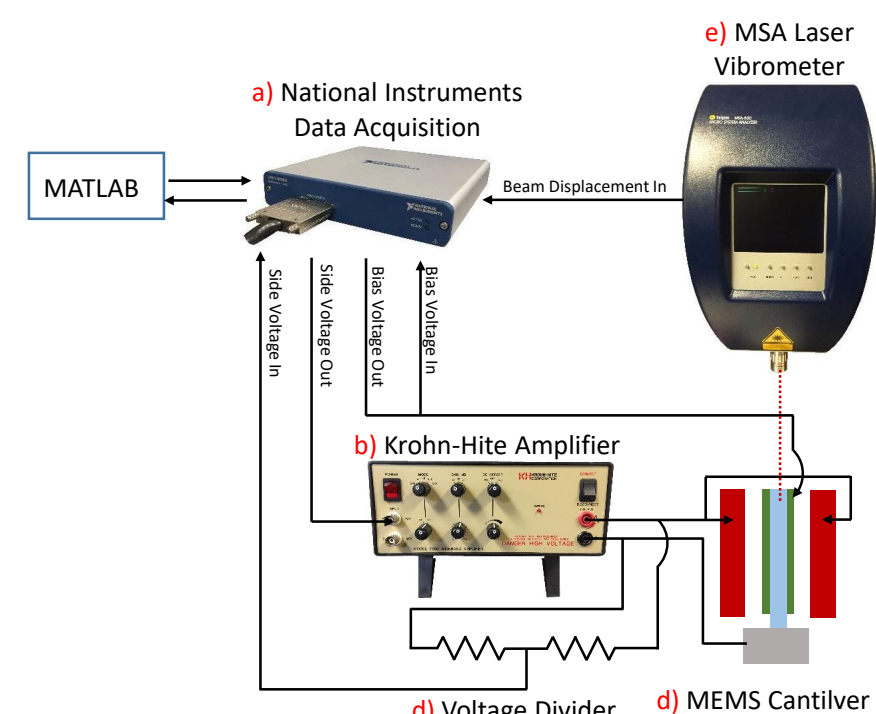

d) Voltage Divider

d) MEMS Cantilver

FIG. 4. Experimental setup

pulled-in position, overcoming both the bias voltage and stiction forces ${ }^{14}$. The model could reach slightly lower voltages before becoming unstable, though this is most likely because the model does not account for the dimples on the underside of the beam. The dimples create an effectively smaller gap between the beam and center electrode, thus increasing the effect of the force from bias voltage, which dominates when the gap is small.

Another interesting result from the static data is the linear relationship between tip deflection and side voltage, especially at higher bias voltages. The slope is approximately $90 \mathrm{~nm}$ per volt on the side electrodes. This 

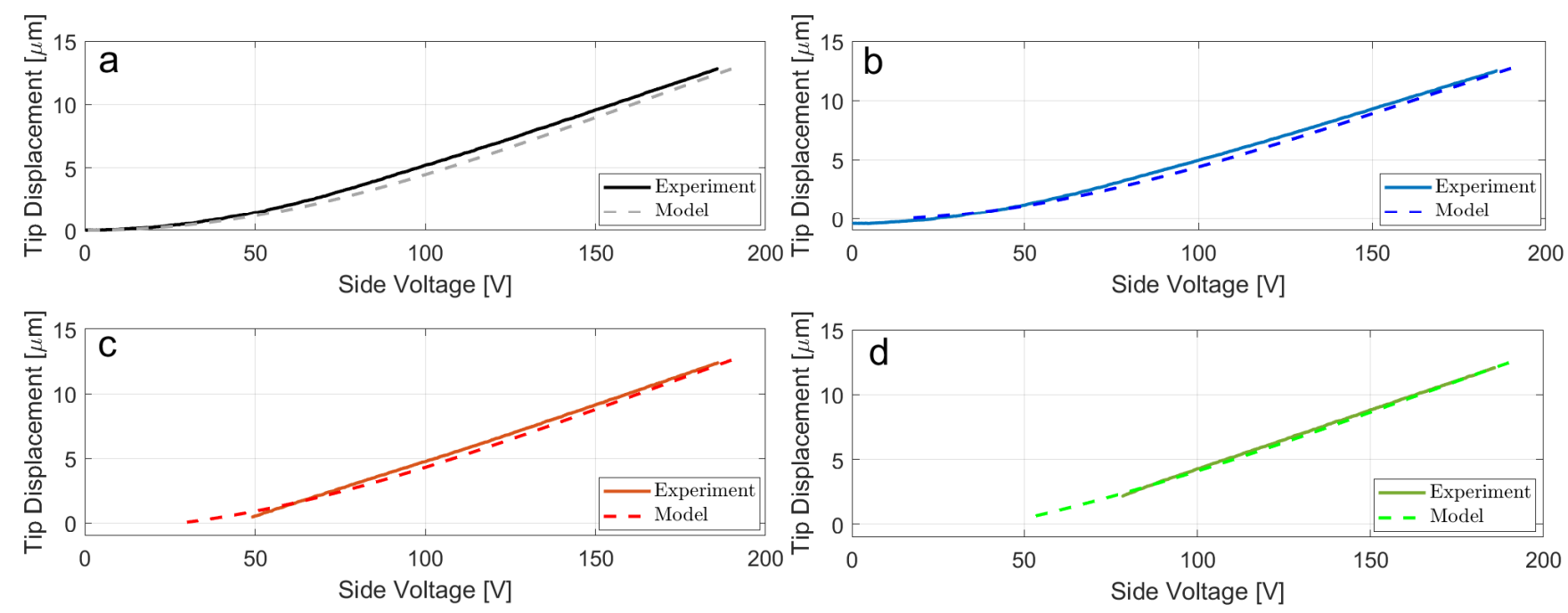

FIG. 5. Comparison of predicted and measured static results for a) $0 \mathrm{~V}$, b) $2 \mathrm{~V}$, c) $4 \mathrm{~V}$, and d) $6 \mathrm{~V}$ bias as a function of side voltage. The results are in very close agreement.

linear relationship is maintained for more than $10 \mu \mathrm{m}$ of static displacement. Because high voltages cannot be applied in devices created using the PolyMUMPs fabrication procedure, $190 \mathrm{~V}$ was not exceeded. However, the tip displacement shows no signs of saturating near 190V and the linear voltage relationship most likely extends much further than the data shows. When there is no bias voltage, an approximately linear relationship exists for deflections above around $4 \mu \mathrm{m}$. However, when $4 \mathrm{~V}$ bias is added to the center electrode, the linearity extends all the way down to $0 \mu \mathrm{m}$. If the bias voltage is too large, such as at $6 \mathrm{~V}$, pull-in will occur at small gaps. Therefore there exists an optimal bias voltage, $4 \mathrm{~V}$, that yields a linear response over the entire static range.

The reason for the linearization between side voltage and tip displacement can be seen by analyzing the force and its associated components. Figure 6 shows the nondimensional forcing components at $6 \mathrm{~V}$ bias as a function of side voltage when evaluated at the static equilibrium points in Figure 5d. As shown in Figure 6a, the total force, which is the sum of each forcing component in Figures $6 \mathrm{~b}-\mathrm{d}$, scales linearly with side voltage. Despite Equation (3) showing the force scaling with the square of the side voltage, when considering the increasing gap, the relationship turns out to be linear. Because the force is now effectively a linear function of side voltage, the solution to Equation (7) will also be a linear function of side voltage.

The reason for the linearization of the force is different when at larger voltages as opposed to smaller voltages. When the side voltage is above $80 \mathrm{~V}$, the $c_{12}$ and $c_{22}$ are negligible compared to the $c_{11}$ component. In this case, the system acts like as if there was just a pure levitation force with no bias voltage. When the side voltage is increased, the upward electrostatic force on the beam also increases. If the beam was completely rigid, this force would scale with the square of the side voltage. But the beam is not rigid and deflects upwards when the side voltage is increased. This deflection increases the gap between the beam and fixed electrodes, which in turn causes the upward electrostatic force to decrease. When the side voltage is large, the decrease in the upward electrostatic force from increasing the gap counteracts the nonlinearly increasing force with voltage and the result is an effectively linear scaling of the total force with side voltage. This can be seen in the static position in Figure $5 \mathrm{a}$, which has no bias voltage, but still shows the linear increase with side voltage. However, when the voltage drops below 80V, Figure 5a shows a nonlinear relationship with side voltage. This is because the levitation force is inherently weak, and unless the voltage is very large, the force is too low to deflect the beam enough for the increasing gap to counteract the scaling of the force with the square of the side voltage.

To achieve linearity at low side voltages, a bias voltage on the center electrode must be used. Comparing the upward levitation $\left(c_{11}\right)$ and downward parallel-plate $\left(c_{22}\right)$ components of the force in Figures $6 \mathrm{~b}$ and $\mathrm{d}$ shows why. As the side voltage decreases, the levitation $c_{11}$ component starts to flatten out around zero. This decrease in total force brings the beam closer to the center electrode and increases the influence of the bias voltage, which pulls the beam downward. The parallel-plate $c_{22}$ component is inversely related to the gap (Equation (6)) and therefore the downward force gets stronger as the gap decreases. When combining the forcing terms, the downward bending of the force from the parallel-plate component counteracts the flattening of the force from the levitation component. Because the parallel-plate force scales with the square of the bias voltage, by choosing 

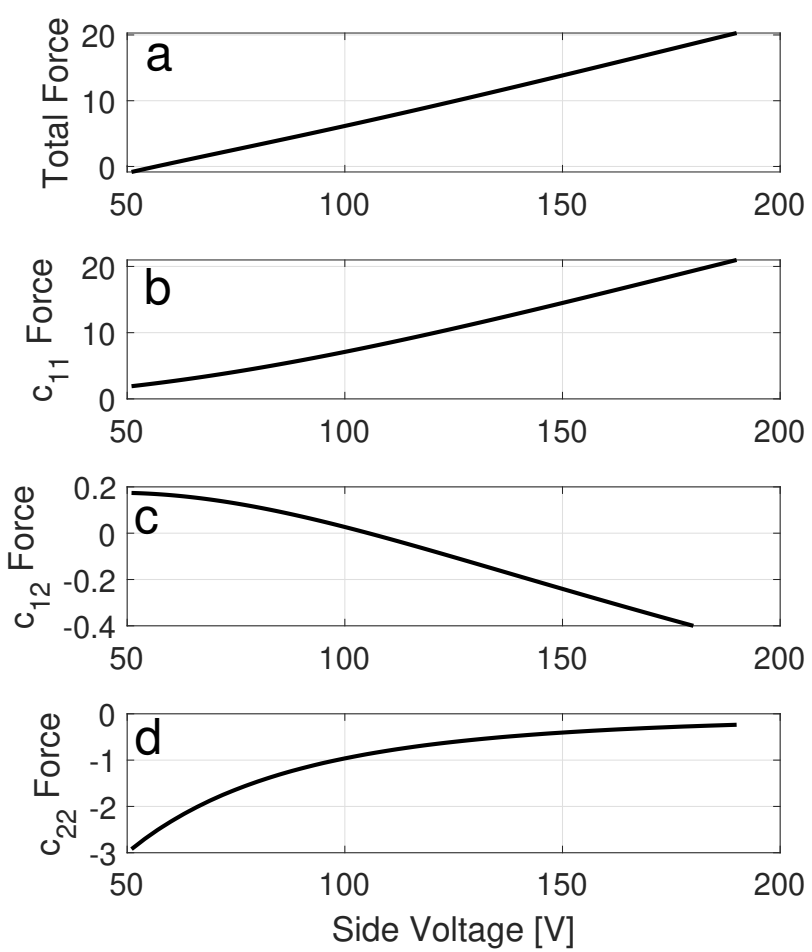

FIG. 6. Nondimensional force at $6 \mathrm{~V}_{\text {bias }}$ as a function of side voltage when evaluated at the static equilibrium position for a) the total force, b) $c_{11}$ component, c) $c_{12}$ component, and d) $c_{22}$ component. Each component is scaled with its associated voltages as denoted in Equation (3).

the appropriate bias, $4 \mathrm{~V}$, the two nonlinear effects cancel each other and the result is a nearly linear force that decreases all the way to zero. If the bias voltage is too large the downward bending from the parallel-plate force overcomes the flattening of the levitation force and the system becomes nonlinear in the negative direction before the force can reach zero and the beam becomes unstable. This is the case for $6 \mathrm{~V}$ bias, where the beam pulls in during the experiment before it can return to its undeflected position. The linearity with side voltage is extraordinarily useful for any actuator that is negatively affected by nonlinearity and requires a large actuating range. Possible applications include micro-mirrors and micro-fluidic devices.

\section{B. Natural Frequency}

Natural frequency is an important property of oscillators and sensors that determines their resolution and sensitivity. To calculate the natural frequency, we must return to the dynamic partial differential equation from Equation (5). Now $w$ becomes a function of time and position so it must be reduced to an ordinary differential equation in time before we can calculate the natural fre- quency. This can be achieved by performing Galerkin's method, which is a modal analysis using separation of variables and integration over $x$ to get a set of secondorder ordinary differential equations that are coupled through nonlinear terms. If just a single mode is to be considered, this will yield a single ordinary differential equation that can be used to calculate the natural frequency.

First, the transverse deflection of the beam is assumed to have separate spatial and time-dependent functions as shown in Equation (8).

$$
w(x, t)=\phi(x) q(t)
$$

The mode shapes, $\phi(x)$, can be solved from the eigenvalue problem, which depends on the boundary conditions. The mode shapes for a simple cantilever are well known and can be expressed by,

$$
\begin{aligned}
\phi_{i}(x)=\cosh \left(\lambda_{i} x\right)-\cos \left(\lambda_{i} x\right)- \\
\sigma_{i}\left(\sinh \left(\lambda_{i} x\right)-\sin \left(\lambda_{i} x\right)\right)
\end{aligned}
$$

where $\lambda_{i}$ is the square root of the $i^{\text {th }}$ nondimensional natural frequency, and $\sigma_{i}$ is a constant. For the first mode, $\phi_{1}$ (herein referred to as $\phi$ ), $\sigma_{1}$ and $\lambda_{1}$ are 0.7341 and 1.875 respectively. However, to account for fabrication imperfections the natural frequency is identified from an experiment and nondimensionalized to use with Equation (9).

After separation of variables, the governing equation is multiplied by $\phi$ and integrated over $\mathrm{x}$ to yield Equation (10), which is a second-order ordinary differential equation that depends only on the time-dependent component of the solution, $q$,

$$
\begin{aligned}
& m \frac{d^{2} q}{d t^{2}}+c \frac{d q}{d t}+k q=V_{\text {side }}^{2} \sum_{i=0}^{9} A_{i} \Phi_{i} q^{i}+ \\
& V_{\text {side }} V_{\text {bias }} \sum_{i=0}^{9} B_{i} \Phi_{i} q^{i}+\frac{1}{2} V_{\text {bias }}^{2} \int_{0}^{1} \phi \frac{\partial c_{22}}{\partial(\phi q)} d x
\end{aligned}
$$

where

$$
\begin{aligned}
m=\int_{0}^{1} \phi^{2} d x \quad c & =c^{*} m \\
k & =\lambda_{1}^{4} m \quad \Phi_{i}=\int_{0}^{1} \phi^{i+1} d x
\end{aligned}
$$

The last term in Equation (10) can be problematic if it is not dealt with appropriately. For the static problem, this term could be fitted with the function given in Equation (6). However, the integration on $\mathrm{x}$ from the modal analysis will create a singularity at $\mathrm{x}=0$, which will make the differential equation impossible to solve. A 
standard parallel-plate has a similar problem; however, it can be mitigated by multiplying the entire equation by the denominator, expanding the polynomial, and then performing Galerkin's method. In this case, two complications prevent that from working. First, the order of the polynomial in the denominator of Equation (6) is not an integer, so it cannot be expanded. Second, multiplying the two 9th order polynomials by a noninteger polynomial will be extraordinarily difficult and tedious later in the problem when solving for the natural frequency. Therefore, this term can be treated numerically as a list of numbers, where linear interpolation is used to estimate the values between data points. This is more computationally expensive, but computation time is not an issue for the natural frequency calculation. Before that can be done, the integration on $\mathrm{x}$ must be performed.

The derivative, $\frac{\partial c_{22}}{\partial(\phi q)}$ can be rewritten using the relationship,

$$
\frac{\partial c_{22}}{\partial q}=\frac{\partial c_{22}}{\partial w} \frac{\partial w}{\partial q}=\frac{\partial c_{22}}{\partial(\phi q)} \frac{\partial(\phi q)}{\partial q}=\frac{\partial c_{22}}{\partial(\phi q)} \phi \frac{\partial q}{\partial q}
$$

which yields,

$$
\frac{\partial c_{22}}{\partial(\phi q)}=\frac{1}{\phi} \frac{\partial c_{22}}{\partial q}
$$

When substituting Equation (13) into Equation (10), the $\phi$ s cancel and the integration becomes 1 . Now that $\mathrm{x}$ has been integrated out of the equation, $q$ represents the deflection of the beam instead of $w$. The derivative of the numerical $c_{22}$ data from COMSOL with respect to the deflection can be substituted in for $\frac{\partial c_{22}}{\partial q}$. The final equation becomes,

$$
\begin{gathered}
m \frac{d^{2} q}{d t^{2}}+c \frac{d q}{d t}+k q=V_{\text {side }}^{2} \sum_{i=0}^{9} A_{i} \Phi_{i} q^{i}+ \\
V_{\text {side }} V_{\text {bias }} \sum_{i=0}^{9} B_{i} \Phi_{i} q^{i}+\frac{1}{2} V_{\text {bias }}^{2} \frac{\partial c_{22}}{\partial q}
\end{gathered}
$$

To find the natural frequency, the eigenvalues of the Jacobian matrix can be calculated by decomposing Equation (14) to two first-order ODEs with $y_{1}=q$ and $y_{2}=\frac{d q}{d t}$, setting the damping coefficient to zero, and plugging the equations into the relationship,

$$
J=\left[\begin{array}{ll}
\frac{\partial \dot{y}_{1}}{\partial y_{1}} & \frac{\partial \dot{y}_{1}}{\partial y_{2}} \\
\frac{\partial \dot{y}_{2}}{\partial y_{1}} & \frac{\partial \dot{y}_{2}}{\partial y_{2}}
\end{array}\right]
$$

which is the Jacobian matrix. The eigenvalues of Equation (15) are,

$$
\Lambda=\sqrt{\frac{V_{\text {side }}^{2} \sum_{i=0}^{9} i A_{i} \Phi_{i} y_{1}^{i-1}+\frac{V_{\text {side }} V_{\text {bias }}}{m} \sum_{i=0}^{9} i A_{i} \Phi_{i} y_{1}^{i-1}+}{\frac{1}{2 m} V_{\text {bias }}^{2} \frac{\partial^{2} c_{22}}{\partial y_{1}^{2}}-\lambda_{1}^{4}}}
$$

where the natural frequency is the imaginary part of the eigenvalue. The only variable that is unknown in this equation is $y_{1}$, which is the beam deflection. The value of $y_{1}$ at each voltage level can be determined from the static model results at the given side and bias voltage, after which the natural frequency can be calculated.

In the experiment, white noise was superimposed on the DC side voltage while holding the bias voltage constant, and the beam tip velocity was recorded with the laser vibrometer. A fast-Fourier transform (FFT) was performed on the velocity signal to extract the natural frequency. Unlike the static experiment, the side voltage was not swept up continuously to $190 \mathrm{~V}$. Instead, the side and bias voltage were kept constant and the natural frequency was extracted at 10 volt intervals. Figure 7 shows the natural frequency results for the experiment and the model.

The model agrees well with the natural frequency experiment. The square root of the natural frequency, $\lambda_{1}$, was 1.7998. The curling of the beam tip in the fabricated beam needs to be incorporated into the model to obtain a better fit with the experiment. Because the beams are very long, residual stress from fabrication causes them to curl upward when released. This increases the gap between the beam and center electrodes and reduces the total electrostatic force. In this case, the beam tip was curled up approximately $2 \mu \mathrm{m}$ out of plane. Ideally, this would be incorporated into both $q$ and the mode shapes. However, the easiest way of addressing this problem is to simply add $2 \mu \mathrm{m}$ of deflection to $q$.

When no bias voltage is applied, Figure 7 shows that the natural frequency is relatively constant for side voltages under approximately 50V. However, at higher bias voltages the natural frequency starts to bend down at low side voltages until the system becomes unstable. This agrees with the initial assessment that the bias voltage is dominant at low gaps because a parallel-plate electrostatic force is correlated with a decrease in natural frequency, while electrostatic levitation increases the natural frequency with higher voltage. In the case of $4 \mathrm{~V}$ and $6 \mathrm{~V}$ bias, the natural frequency can be tuned from less than $6 \mathrm{kHz}$ up to almost $12 \mathrm{kHz}$. This is an increase of almost $100 \%$, which gives the beam a tremendous amount of tunability to control where the first resonant peak is located. Many devices, such as sensors, operate near their natural frequency to boost the signal-to-noise ratio. This electrostatic tunability enables accounting for any fabrication imperfection or environment operation changes to ensure a large signal-to-noise ratio and resolution. The 

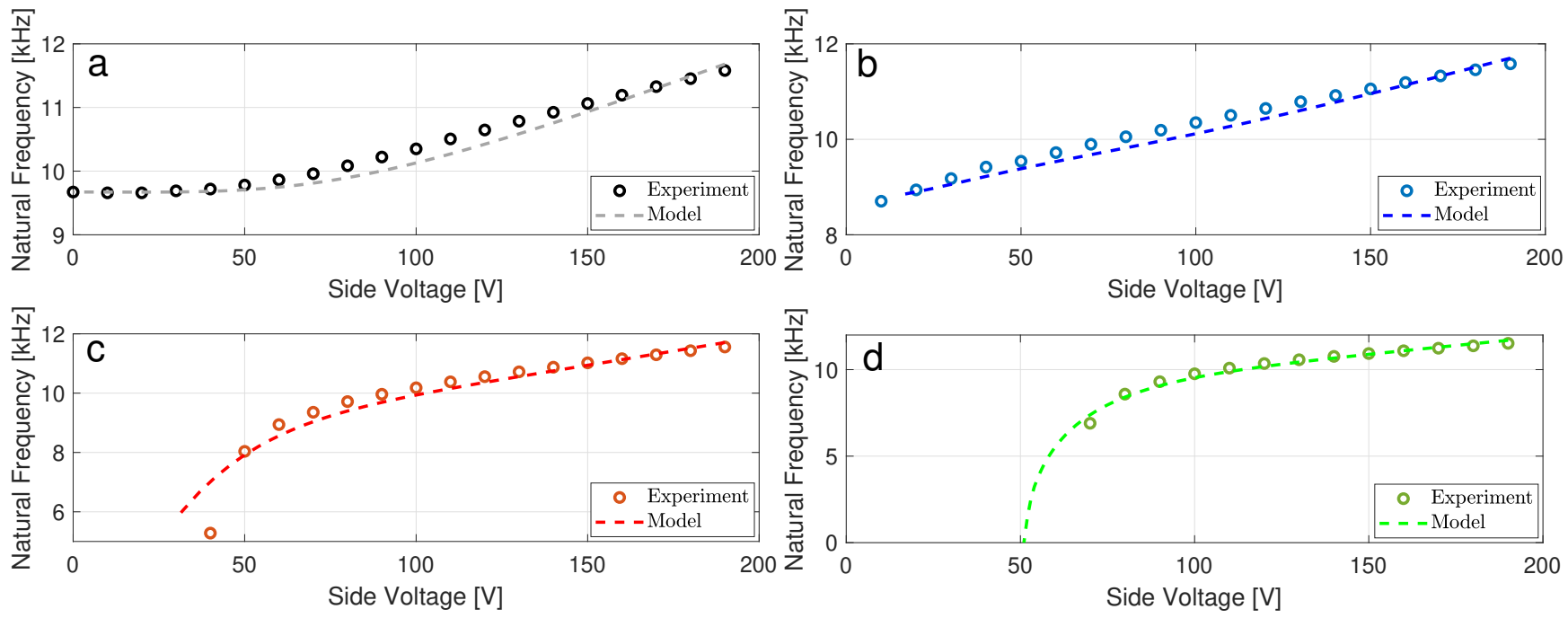

FIG. 7. Natural frequency results for a) $0 \mathrm{~V}$, b) $2 \mathrm{~V}$, c) $4 \mathrm{~V}$, and d) $6 \mathrm{~V}$ bias as a function of side voltage.

proposed system can be tuned to capture a wide range of frequencies, which is highly desirable in many sensors.

At an optimum value of the bias voltage, the highest linearity of natural frequency and the side voltage is achieved. If the bias voltage is set to approximately $2 \mathrm{~V}$, the downward bending of the natural frequency curve at low side voltage reaches a point where it matches the slope at higher side voltages. This creates a linear shift in natural frequency with side voltage. Unlike the static solution, the natural frequency is related to the slope of the force at the equilibrium position instead of the magnitude. The slope of the force is the linear stiffness, which is related to the natural frequency through the relationship,

$$
\omega_{n}=\sqrt{\frac{k_{e f f}}{m}}
$$

where $\omega_{n}$ is the natural frequency, $m$ is the mass, and $k_{e f f}$ is the effective stiffness, which is the sum of the mechanical stiffness of the beam $\left(k_{\text {mech }}\right)$ and the added stiffness from the electrostatic force $\left(k_{\text {elec }}\right)$, where $k_{\text {elec }}$ is much larger than $k_{m e c h}$. Because $m$ and $k_{\text {elec }}$ are constant, $k_{\text {elec }}$ must be related to the side voltage. If $\omega_{n}$ is linearly related to side voltage, then $k_{\text {elec }}$ must be related to the square of the side voltage. This is confirmed to be true by analyzing the slope of the force at each static equilibrium position. The linear relationship in frequency is approximately $16 \mathrm{~Hz}$ per volt and is maintained for more than $2.9 \mathrm{kHz}$ of frequency shift. The combination of parallel-plate and electrostatic levitation enables the designers to have a wider range of capabilities that is not possible with each mechanism on its own.

\section{Frequency Response}

To obtain the model frequency response, the dynamic equation of motion must be solved. Much of the procedure for calculating the natural frequency of Equation (5) can be used for the frequency response as well. Equation (14) can be integrated directly using linear interpolation to determine $\frac{\partial c_{22}}{\partial q}$. However, the computation expense of using linear interpolation becomes problematic and creates very long solve times. To reduce the integration time, $\frac{\partial c_{22}}{\partial q}$ can be fit with the inverse non-integer polynomial in Equation (6) with $q$ replacing $w$. This is not problematic anymore because the substitution is occurring after the modal analysis, which is where the problems were occurring initially. This significantly reduces the computation time so that the frequency response can be calculated. The final dynamic equation is given in Equation (18).

$$
\begin{aligned}
& m \frac{d^{2} q}{d t^{2}}+c \frac{d q}{d t}+k q=V_{\text {side }}^{2} \sum_{i=0}^{9} A_{i} \Phi_{i} q^{i}+ \\
& V_{\text {side }} V_{\text {bias }} \sum_{i=0}^{9} B_{i} \Phi_{i} q^{i}-V_{\text {bias }}^{2} \frac{0.4626}{\left(q+\frac{d}{h_{3}}\right)^{2.15}}
\end{aligned}
$$

The damping coefficient is estimated from the experiment, which was conducted at approximately 300mTorr, which correlates to a quality factor of 500 . The quality factor is used in Equation (18) by the relationship $c=\lambda_{1}^{2} / Q$.

To conduct the experiment, the side voltage is given an AC voltage on top of the DC offset. The DAQ supplies the AC voltage, and the DC offset function of the KrohnHite amplifier applies the DC side voltage. The bias was 
held constant at the same $0 \mathrm{~V}, 2 \mathrm{~V}, 4 \mathrm{~V}$, and $6 \mathrm{~V}$ values used in the static and natural frequency tests. For the frequency response, the tip velocity was measured with the vibrometer. Figure 8 shows the frequency response around the first natural frequency at $0 \mathrm{~V}, 2 \mathrm{~V}, 4 \mathrm{~V}$, and $6 \mathrm{~V}$ bias, with $170 \mathrm{VDC}$ and $0.5 \mathrm{VAC}$ on the side electrodes.

As with the static and natural frequency results, the frequency response model agrees with the experiment. In the experiment, the backsweep branch continues to grow until it starts tapping on the center electrode and falls back down to the lower branch. If the bias voltage is high enough, instead of falling back down to the lower branch, it pulls in, as is the case at $6 \mathrm{~V}$ bias. Before pull-in occurs, the backsweep branch becomes very flat and increases in size. This extension of higher branch oscillation is because of the quadratic nonlinearity that causes excessive softening from electrostatic levitation and parallelplate schemes. With no bias, the backsweep branch ends around $11 \mathrm{kHz}$, but with $6 \mathrm{~V}$ bias the branch travels back to $10 \mathrm{kHz}$ before pulling in. The high amplitude vibration could be useful for the development of high-resolution oscillators. The triggering of dynamic pull-in near the resonant peak can help create combined systems that act as a sensor and a switch, which triggers when the sensed quantity increases past a threshold. The levitation force can be used to release the pulled-in structure and reuse the device. To get more insight into the motion of the beam, the model displacement was calculated and is shown in Figure 9.

This dynamic displacement reaches upward of $28 \mu \mathrm{m}$ with no bias voltage. Increasing the bias bends the peak down and to the left, which drops the peak amplitude by about $3 \mu \mathrm{m}$ for $6 \mathrm{~V}$ bias. Unlike static and natural frequency, the addition of the bias voltage always increases the nonlinearity of the system. This is because levitation and parallel-plate electrostatic forces create softening in the frequency response, therefore the combination of the two yields more softening. Therefore, MEMS oscillators and resonators that require a linear frequency response would not benefit from this method.

However, other applications that are less hindered by the nonlinear frequency response may be able to exploit other behaviors of the combined system, such as dynamic pull-in shown in Figure 8d. One possible application could be a pressure sensor that triggers dynamic pullin at the resonant peak when the pressure drops below a threshold value. As reported in ${ }^{12}$, the resonance region of the levitation actuator is heavily dependent on the ambient pressure because the oscillation amplitude is very large and squeeze film damping effects are significant. The voltage at which dynamic pull-in occurs could be related to the ambient pressure to create a threshold pressure sensor that utilized the switching mechanism proposed in ${ }^{14}$.

Numerous other applications that exploit this property could be conceived for this device. The large linear stroke and tunability of the natural frequency can inspire the creation of a variety of micro-actuators or manipulators in biomedical devices.

\section{CONCLUSION}

In this study, it was demonstrated theoretically and experimentally that merging parallel-plate and electrostatic levitation actuators can yield increased functionality of MEMS actuators. The relationship between static tip displacement and natural frequency with side voltage can be linearized by choosing an appropriate bias voltage, which can greatly simplify and minimize the electronic circuitry for processing information from sensors and controlling actuator motion. The natural frequency can be tuned from $6 \mathrm{kHz}$ up to $12 \mathrm{kHz}$, increasing tunability, which gives sensors a larger spectrum of detectable frequencies with large signal-to-noise ratios. The softening branch of the frequency response can be flattened and extended by adding a larger bias voltage. The larger bias also trigger dynamic pull-in near the resonant peak, which is promising for combined sensors and switches that use pull-in to close a circuit. The levitation force enables the system to release from its pulled-in position, allowing the combined sensor and switch system to be more reliable as it can be reused many times without failure. While parallel-plate and electrostatic levitation MEMS have a tremendous amount of advantages individually, by combining these two systems, more advanced and efficient MEMS can be created.

\section{ACKNOWLEDGMENT}

This work was funded by the NFS ECCS grant no. 1608692 .

${ }^{1}$ M. I. Younis, Mems Linear and Nonlinear Statics and Dynamics (Springer, New York, 2011).

${ }^{2}$ A. Alneamy, M. Al-Ghamdi, S. Park, M. Khater, E. AbdelRahman, and G. Heppler, Journal of Applied Physics 125, 024305 (2019).

${ }^{3}$ M. Al-Ghamdi, M. Khater, and E. Abdel-Rahman, Applied Physics Letters 113, 153501 (2018).

${ }^{4}$ M. A. Rosa, M. A. Bruyker, A. R. Volkel, E. Peeters, and

J. Dunec, Journal of Micromechanics and Microengineering 14, 446 (2004).

${ }^{5}$ Q. Chen, Y.-C. Lai, J. Chae, and Y. Do, Physical Review B 87, 144304 (2013).

${ }^{6}$ Y. Linzon, B. Ilic, S. Lulinsky, and S. Krylov, Journal of Applied Physics 113 (2013), 10.1063/1.4802680.

${ }^{7}$ R. N. Miles, IEEE Sensors 18, 5691 (2018).

${ }^{8}$ K. B. Lee and Y. H. Cho, Journal of Microelectromechanical Systems 10, 128 (2001).

${ }^{9} \mathrm{~S}$. He and R. Ben Mrad, IEEE Transactions on Industrial Electronics 52, 974 (2005).

${ }^{10} \mathrm{C}$. Fan and S. He, Journal of Microelectromechanical Systems 24, 2049 (2015).

${ }^{11}$ M. Pallay, M. Daeichin, and S. Towfighian, Nonlinear Dynamics 89, 1525 (2017).

${ }^{12}$ M. Pallay and S. Towfighian, Sensors and Actuators A: Physical 277, 134 (2018).

${ }^{13}$ M. Ozdogan, M. Daeichin, A. Ramini, and S. Towfighian, Sensors and Actuators A Physical, 265, 20 (2017). 

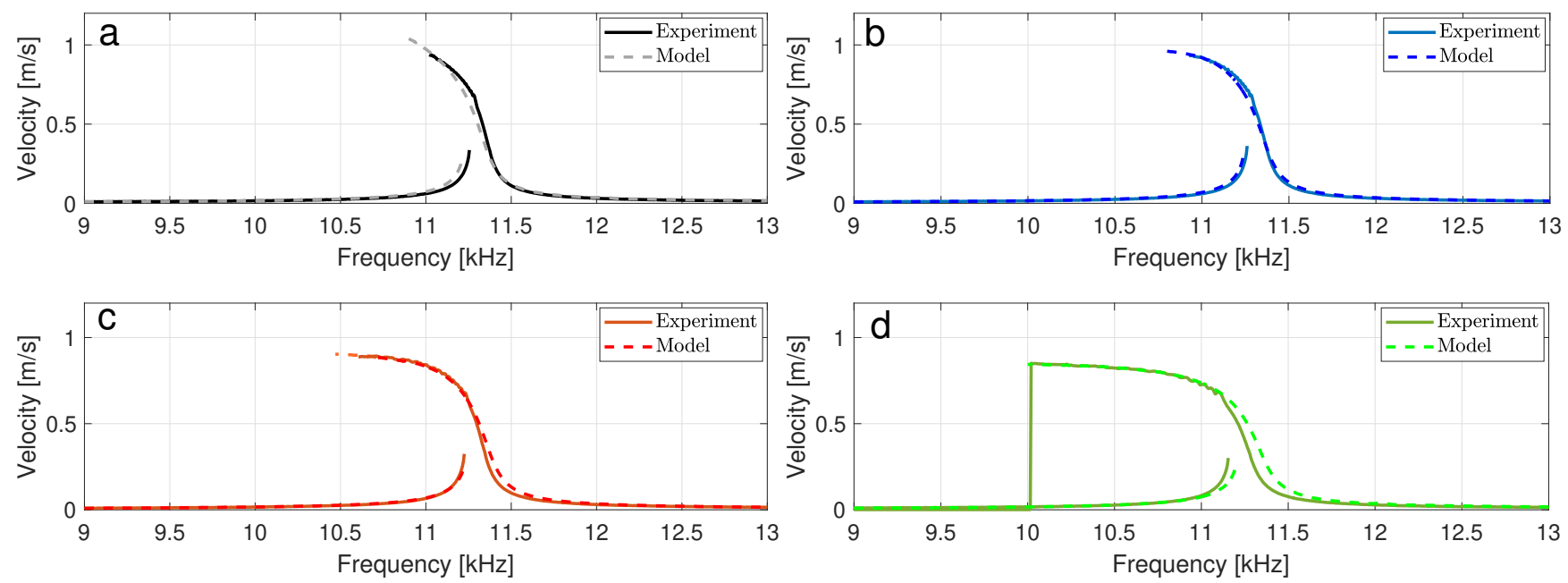

FIG. 8. Frequency response around the first natural frequency for a) $0 \mathrm{~V}, \mathrm{~b}) 2 \mathrm{~V}$, c) $4 \mathrm{~V}$, and d) $6 \mathrm{~V}$ bias at $170 \mathrm{VDC}$ and $0.5 \mathrm{VAC}$ on the side electrodes.

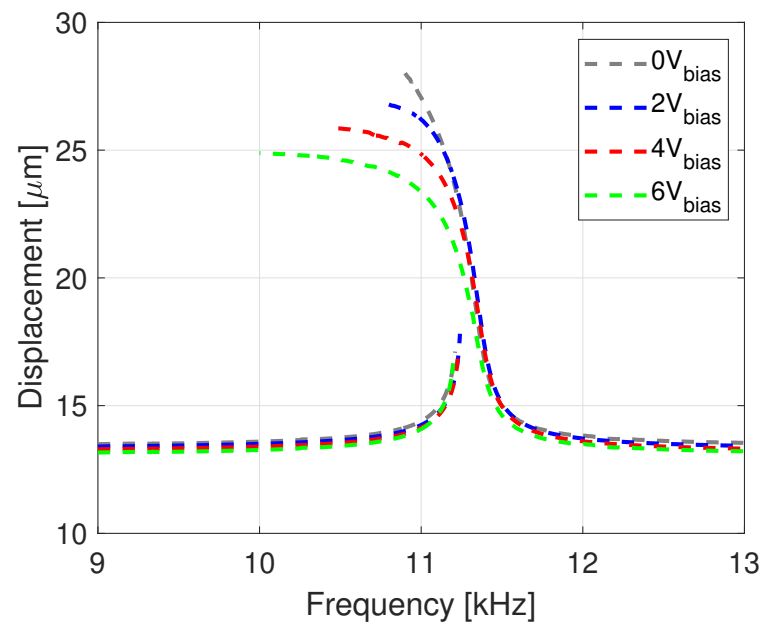

${ }^{14}$ M. Pallay and S. Towfighian, Applied Physics Letters 113, 213102 (2018).

${ }^{15}$ A. Cowen, B. Hardy, R. Mahadevan, and S. Wilcenski, "PolyMUMPs Design Handbook a MUMPs@ process," (2011).

FIG. 9. Model displacement frequency response around the first natural frequency for $0 \mathrm{~V}, 2 \mathrm{~V}, 4 \mathrm{~V}$, and $6 \mathrm{~V}$ bias at $170 \mathrm{VDC}$ and $0.5 \mathrm{VAC}$ on the side electrodes. 